\title{
Venous Thromboembolism
}

National Cancer Institute

\section{Source}

National Cancer Institute. Venous Thromboembolism. NCI Thesaurus. Code C99537.

Occlusion of the lumen of a vein by a thrombus that has migrated from a distal site via the blood stream. 